\title{
Improved Preservation Technology for Non-Instantaneous Deteriorating Inventory Using Boundary Condition Estimation
}

\author{
Ihsan Hishamuddin*, Siti Suzlin Supadi", Mohd Omar \\ Institute of Mathematical Sciences, University of Malaya, Kuala Lumpur, Malaysia \\ Email address: \\ sma170044@siswa.um.edu.my (I. Hishamuddin), suzlin@um.edu.my (S. S. Supadi), mohd@um.edu.my (M. Omar) \\ ${ }^{*}$ Corresponding author
}

To cite this article:

Ihsan Hishamuddin, Siti Suzlin Supadi, Mohd Omar. Improved Preservation Technology for Non-Instantaneous Deteriorating Inventory Using Boundary Condition Estimation. Applied and Computational Mathematics. Vol. 9, No. 4, 2020, pp. 118-129.

doi: 10.11648/j.acm.20200904.12

Received: June 10, 2020; Accepted: June 30, 2020; Published: July 17, 2020

\begin{abstract}
Various forms of preservation technology nowadays allow businesses to handle valuable perishable items with greater flexibility. Even with a wide variety of preservation techniques, the mathematical modelling of its implementation in EOQ literature remains rigid. The paper aims to integrate an improved preservation technology in a non-instantaneous deteriorating inventory model for businesses maximizing their average total cycle profit. The improved preservation technology furthers the delay to the time within the cycle where deterioration begins and enhances the durability of inventory that allows operators to employ a less prudent holding facility. Another improvement in this area is the accurate accumulation of preservation cost depending on the inventory level at hand. The conventional EOQ method of forming the objective function before choosing the optimal values for our two decision variables (Cycle time and level of preservation) is undertaken. The cycle time is split in two, differing in their inventory process (deterioration beginning in the second period). The time when deterioration begins is derived using the model's boundary conditions, a first attempt within the area. The optimal solution set is solved for a numerical example using an algorithm to demonstrate the model and prove the global nature of the solution. An investigation into the gains from the improved preservation technology is conducted by dissecting the effects within each individual component within the objective function. 3 separate channels by which this improved preservation technology modelling benefits the business model is found namely shifting to the higher profitable period, effects towards preservation affected costs and the returns to scale from successively increasing preservation levels. Sensitivity analysis is conducted to demonstrate and confirm the findings. The paper discovers great benefits from such an improved modelling that warrants further attention within the scope of preserved inventory models, especially on how levels of preservation could influence the traditional decision variable optimized such as cycle time or ordering frequency. Findings of the paper would have significant benefits to different inventory models with its own delay before deterioration and holding facility requirement.
\end{abstract}

Keywords: Operational Research, Mathematical Modelling, Inventory, Preservation, Non-Instantaneous

\section{Introduction}

Deterioration within inventory occurs in various industries such as imaging, health sector and perishable food-items. Due to its universal relevance, companies employ preservation technologies with varying intensity to control the deterioration rate and lower losses owing to inventory loss via deterioration. Traditionally, preservation technology has been treated merely as a machinery installment incurring constant cost per time during the operation cycle $[10,18,21$, 36]. This approach has been adopted by nearly all papers that involves a utilization of preservation technology $[12,13,20$, $28,35]$. While this approach is straightforward to model as merely a multiple of the total cycle time, a shortcoming from this approach is the assumed constant preservation cost independent of the inventory level at hand.

Realistically, this treatment neglects the varying inventory levels held during the cycle that would require varying 
amounts of preservation. For example, one cannot expect a single air conditioning unit to effectively preserve 1 tonne of perishable fruits as well as it preserves $10 \mathrm{~kg}$ of the same item. Therefore a more realistic preservation technology would incur costs depending on the amount of time that it is being employed and also depending on the amount of inventory it preserves. An improvement from the previous assumption would enable inventory operators the flexibility to incur preservation costs according to the size of inventory at hand for each time period. This type of Inventory Proportional Preservation Cost has never been adopted in any previous paper.

Within the scope of deteriorating inventory models, literature can be categorized by the start of the deterioration process within the cycle. Earlier papers that did not consider preservation adopted the straightforward approach where deterioration begins at the start of the cycle $[1,3,8,9,19,23$, $31,32,33]$. This approach has been adopted by recent studies that now consider preservation to affect the deterioration process $[4,6,7,12,20,22,29]$. These types of deterioration process are commonly known as Instantaneously Deteriorating inventory. This might apply to some very delicate and fragile inventory that begins to deteriorate the moment it is stored such as radioactive isotopes or oil paintings. Currently however, this type of deterioration is rare since majority of perishable inventory would take a while before it starts being spoilt fully beyond consumption quality. As an alternative, a delay before deterioration begins affecting the inventory process during the cycle time is considered. For example, deterioration could begin as inventory is transferred from the factory to the warehouse [2, 5]. These types of inventory are classified as NonInstantaneously Deteriorating Inventory models [11, 14, 25, 34]. Although this is an improved and more realistic assumption, majority of papers do not elaborate on how the delay is determined $[13,15,16,26,28]$. Usually the delay before deterioration begins is taken as an exogenously determined factor not affected by the decision variables as summarized in table 1 below:

Table 1. Approach for Start of Deterioration in Recent Literature.

\begin{tabular}{llll}
\hline Paper & Instantaneous & Exogeneously Determined Delay & Endogeneously Controllable Delay \\
\hline Tao et. al (2019) & $\boldsymbol{V}$ & \\
Dye and Yang (2017) & $\checkmark$ & \\
Mishra (2014) & & $\checkmark$ \\
Yang et. al (2018) & & \\
Zhang et. al (2015) & & \\
Singh, Mishra and Pattanayak (2017) & & \\
Mishra, Wu, Tsao and Tseng (2019) & $\checkmark$ & \\
Maihami and Karimi (2014) & & \\
Li, He, Zhou and Wu (2019) & $\checkmark$ & \\
Singh and Sharma (2013) & $\boldsymbol{V}$ & \\
Huang, He and Li (2018) & & \\
\hline
\end{tabular}

With various preservation technologies such as refrigeration, pesticide and handling instruments, it is surprising that preservation has not been seriously considered as a decision variable that could affect the delay before preservation $[17,24,25,29,38]$. Up to date only one paper addressed the delay period within their model, where the delay is affected by the preservation level though it was not explicitly derived and described [30]. Suggestively, a higher preservation level would further delay the time before deterioration process begins within the time cycle. This would occur since higher preservation levels would decrease the perishability of inventories.

Finally, the implementation of preservation technologies could also give positive externalities within the Model that benefits the operators. These benefits could come with the form of reducing related costs such as emission penalties or reduced inventory holding costs as found commonly in inventory models with green initiatives to conserve the environment $[15,27,29,34]$. These positive benefits could also come from tax leeway given by certain countries for operators that adopt preservation technologies. In other cases, perishable products that become more durable with preservation could be held in less delicate and expensive storage facilities, lowering the inventory holding cost.
The paper starts by deriving the estimate equations for the initial period where no deterioration is in effect and the second period where deterioration is present. Using the estimate equations from both periods, the point within the cycle where deterioration begins $\left(t_{0}\right)$ is derived. This point within the cycle time $\left(t_{0}\right)$ is further explored through its expression. Following on, the objective function is constructed to be maximised. The order conditions for optimality are then derived for the two decision variable which is the length of cycle for the second period $(T)$ and also the level preservation $(\beta)$. We then solve a numerical example to find the solution for a given scenario using a simple algorithm. The optimal decision variable values are then verified as the global solution using the Hessian matrix. To thoroughly investigate the advantages of employing preservation technology in deteriorating inventories, the gains in profit from implementing this improved preservation technology are deconstructed. The individual terms are examined to specify by which channels preservation technology may lead to an increase in profit or avoid loss. Next, some sensitivity analysis is conducted to verify the outcomes found. Finally, improvements to be considered in future research are suggested before concluding remarks are made on the outcomes from this research. 


\section{Notations and Assumptions}

\subsection{Notations}

Table 2. Description of notations used in the paper.

\begin{tabular}{ll}
\hline Notation & Description \\
\hline $\mathrm{I}_{1}(\mathrm{t}), \mathrm{I}_{2}(\mathrm{t})$ & Inventory level for each period \\
$\mathrm{t}$ & Current time within each period \\
$\mathrm{t}_{0}$ & First period cycle time \\
$\mathrm{T}$ & Second period cycle time \\
{$\left[0, \mathrm{t}_{0}\right]$} & First period cycle range \\
{$[0, \mathrm{~T}]$} & Second period cycle range \\
$\mathrm{Q}$ & Inventory level at start \\
$\alpha$ & Initial deterioration rate \\
$\beta$ & Preservation level \\
$\theta=\frac{\alpha}{1+\beta}$ & Effective deterioration rate \\
$\lambda$ & Inventory proportional demand rate \\
$\mathrm{D}$ & Constant demand rate in period 2 \\
$\mathrm{OC}$ & Ordering cost/unit \\
$\mathrm{DC}$ & Deterioration cost/unit \\
$\mathrm{HC}(\beta)$ & Holding cost/unit/time \\
$\mathrm{PC}(\beta)$ & Preservation cost/unit/time \\
$\mathrm{I}_{1}^{\mathrm{T}(\beta)}$ & Total inventory held in first period with preservation \\
$\mathrm{I}_{2}^{\mathrm{T}(\beta)}$ & Total inventory held in second period with preservation \\
\hline
\end{tabular}

$I(t)$ - Inventory Level

\subsection{Assumption}

There are now two separate periods $\left(0<t_{1}<t_{0}\right)$ and $(0<$ $t_{2}<T$ ) with two different inventory processes. Deterioration takes into effect from the end of the first period $\left(t_{0}\right)$ or the start of the second period, where the inventory process changes from Eq. (1) to Eq. (3).

Preservation technology enters as a discounting factor as shown in (4) that reduces the proportion of deterioration, with diminishing marginal returns. No shortages are allowed during both cycle periods $\left[I_{1}(t), I_{2}(t)>0\right]$. Total demanded and deteriorated inventory is less than the amount of inventory available at any point in the cycle time $[(I(t) *(\lambda+\alpha))+D<1]$.

Ordering Cost per unit applies to each unit ordered initially at the start of the cycle $\left(O C=C_{o}\right)$. Deterioration Cost per unit equals revenue lost had the inventory been sold $\left(D C=C_{d}=P\right)$. Preservation Cost per time-inventory depends on the level of preservation employed, $\left[P C(\beta)=\left(C_{p}\right)^{*}(\beta)\right]$ when $[\beta>0]$, and $[P C=0]$ when $[\beta=0]$. Preservation investment discounts per time-inventory holding cost depending on the level of preservation when employed, $\left[H C(\beta)=\left(C_{h}\right) /(\beta)\right]$ when $[\beta>0]$, and $\left[H C(\beta)=C_{h}\right]$ when $[\beta=0]$.

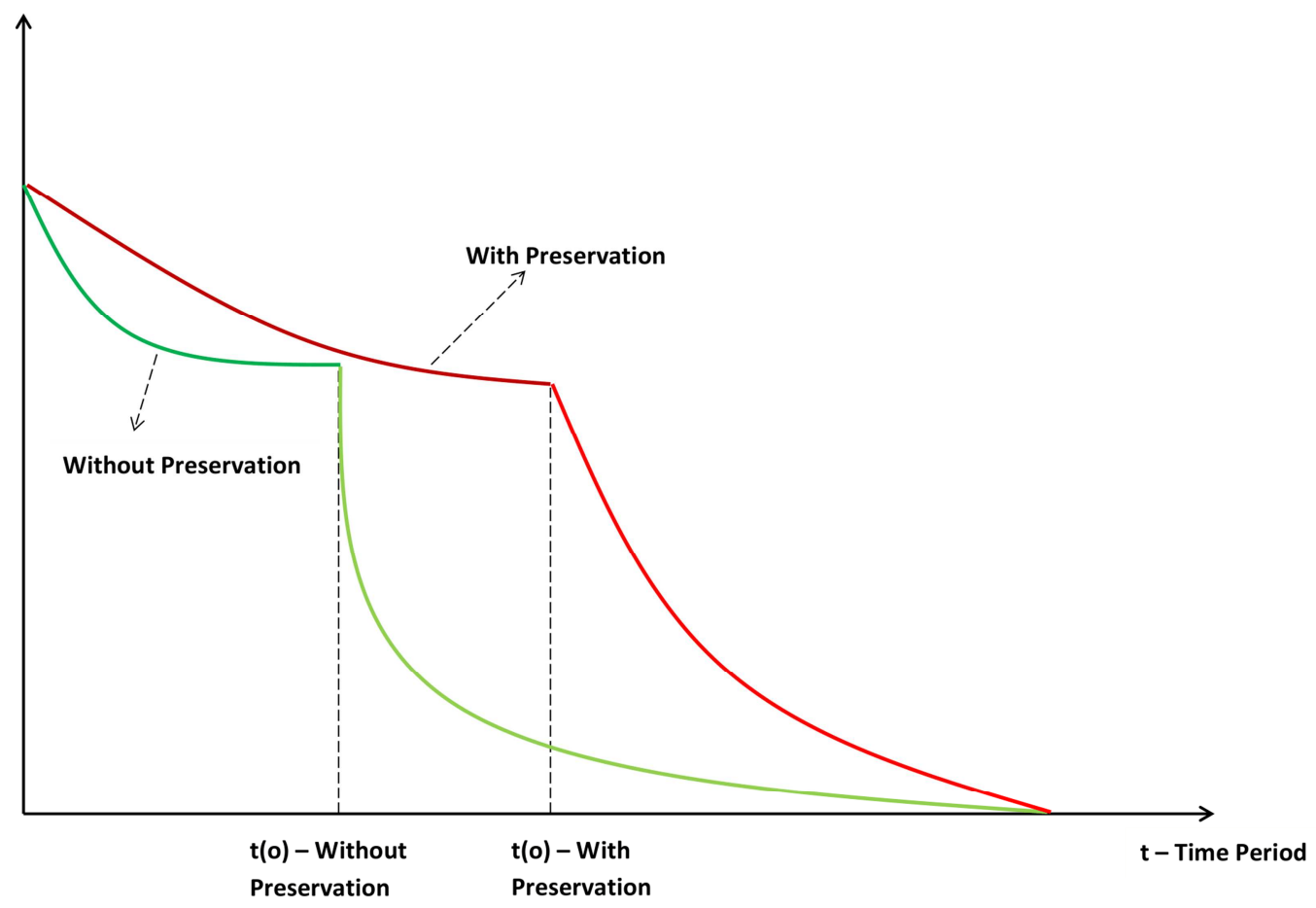

Figure 1. Inventory Process With and Without Preservation Technology Implementation.

\section{Inventory Process}

\subsection{Period $1\left(0<t_{1}<t_{0}\right)$}

In the first period, there are no deterioration and the demand function consists of only a proportional demand $(\lambda)$ dependent on the current inventory level at hand:

$$
\frac{d I_{1}(t)}{d t}=-\lambda I_{l}(t)
$$

Solving the differential equation above with the boundary condition of $(I=Q)$ at $\left(t_{l}=0\right)$ gives estimation for Inventory level in the first period:

$$
I_{l}(t)=\frac{Q}{\exp (\lambda t)}
$$

\subsection{Period $1\left(0<t_{2}<T\right)$}

In the second period, effective deterioration $(\theta)$ is now in effect as a proportion of current inventory level at hand 
$\left(\theta I_{l}(t)\right)$ while demand now includes a small constant amount (D) to ensure there is still demand even with minimal levels of inventory:

$$
\frac{d I_{2}(t)}{d t}=-D-\lambda I_{1}(t)-\theta I_{1}(t)
$$

Depending on the level of preservation technology used, effective deterioration is reduced at a diminishing marginal rate (Lemma 1). We can check this property by the partial differential of the effective deterioration rate with respect to the preservation level.

$$
\begin{aligned}
\theta(\beta)= & \frac{\alpha}{1+\beta} \\
& \frac{\partial \theta}{\partial \beta}=\frac{-\alpha}{(1+\beta)^{2}}, \frac{\partial^{2} \theta}{\partial \beta^{2}}=\frac{2 \alpha}{(1+\beta)^{3}}
\end{aligned}
$$

Solving the differential equation above with the boundary condition of $(I(T)=0)$ at $\left(t_{2}=T\right)$ gives the estimation for Inventory level during the second period:

$$
I_{2}(t)=\frac{D}{\lambda+\theta}[\exp ((\lambda+\theta)(T-t))-1]
$$

\subsection{Start of Deterioration $\left(t_{0}\right)$}

Equating $I_{1}\left(t_{0}\right)$ with $I_{2}(0)$ as per our assumption on the two time periods gives out the expression of $\left(t_{0}\right)$ that ensures the continuity and continuity and consistency from the first period to the second period:

$$
\begin{gathered}
I_{1}\left(t_{0}\right)=I_{2}(0) \text { or } \frac{Q}{\exp (\lambda t 0)}=\frac{D}{\lambda+\theta}[\exp ((\lambda+\theta) T)-1] \\
t_{0}=\frac{1}{\lambda} \ln \left[\frac{Q(\lambda+\theta)}{[\exp ((\lambda+\theta) T)-1] D}\right]
\end{gathered}
$$

This expression gives the point within the cycle time where deterioration kicks in or the length of the first period within our cycle. Thus after $\left(t_{0}\right)$, the inventory process and estimate changes from Eq. (2) to Eq. (6). As seen within the expression, $\left(t_{0}\right)$ depends on the length of second period cycle time $(T)$ and preservation $(\beta)$. Checking the first order condition lets us check how the decision variables affect $\left(t_{0}\right)$ :

$$
\begin{gathered}
\frac{\partial t_{0}}{\partial T}=\frac{1}{\lambda}\left[\frac{(\lambda+\theta) \exp ((\lambda+\theta) T)}{[1-\exp ((\lambda+\theta) T)]}\right] \\
\frac{\partial t_{0}}{\partial \beta}=\frac{\alpha}{\lambda(1+\beta)^{2}}\left\{\frac{T \exp ((\lambda+\theta) T)}{[\exp ((\lambda+\theta) T)-1]}-\frac{(\lambda+\theta)}{1}\right\}
\end{gathered}
$$

We can prove that employing a higher preservation level reduces effective deterioration rate $(\theta)$ which prolongs the delay (length of first period) before deterioration kicks-in (Lemma 2):

$$
t_{0}^{(\beta>0)}>t_{0}^{(\beta=0)}
$$

when $t_{0}{ }^{(\beta>0)}=\frac{1}{\lambda} \ln \left[\frac{Q(\lambda+\theta)}{[\exp ((\lambda+\theta) T)-1] D}\right], \theta(\beta)=\frac{\alpha}{1+\beta}$

Proof: The statement to be proven is, $t_{0}$ increases as $\theta$ decreases or as $\beta$ increases.

We impose the condition for $\left(t_{0}>0\right)$ requiring the terms in the early bracket to be positive. We observe the behavior of the term within the curly bracket as $(\beta)$ and thus $(\theta)$ changes.

The numerator term of $Q(\lambda+\theta)$ rises linearly while the term in the denominator $[\exp ((\lambda+\theta) T)-1] D$ rises exponentially as $(\theta)$ increases. Therefore the term in the curly bracket and thus $\left(t_{0}\right)$ decreases as $(\theta)$ increases. This would occur when $(\beta)$ decreases.

Conversely this would prove our statement and Lemma 2 to be proven.

\subsection{Inventory Held During Cycle}

The total inventory held during the first period could be obtained by integrating the first period inventory estimate over the time cycle of the first period:

$$
\int_{0}^{t_{0}} I_{1}(t) d t=\frac{Q}{\lambda}\left[1-\frac{1}{\exp \left(\lambda t_{0}\right)}\right]=I_{1}^{T(\beta)}
$$

It can be proven that inventory held in the first period is greater with (higher) use of preservation technology (Lemma 3).

Proof: From the expression above, an alternate first period inventory held with no (lower) preservation is as below:

$$
\begin{aligned}
& I_{l}^{T(\beta)}=\int_{0}^{t_{0}} I_{l}(t)^{(\beta>0)}(t) d t=\frac{Q}{\lambda}\left[1-\frac{1}{\exp \left(\lambda t_{0}(\beta>0)\right)}\right], \\
& I_{l}^{T(0)}=\int_{0}^{t_{0}} I_{l}(t)^{(\beta=0)}(t) d t=\frac{Q}{\lambda}\left[1-\frac{1}{\exp \left(\lambda t_{0}(\beta=0)\right)}\right]
\end{aligned}
$$

Since via Lemma 2:

$$
\mathrm{t}_{0}^{(\beta>0)}>\mathrm{t}_{0}^{(\beta=0)}
$$

It can be clearly seen:

$$
\frac{Q}{\lambda}\left[1-\frac{1}{\exp \left(\lambda t_{0}(\beta>0)\right)}\right]>\frac{Q}{\lambda}\left[1-\frac{1}{\exp \left(\lambda t_{0}(\beta=0)\right)}\right] .
$$

The total inventory held during the second period could be obtained by integrating the inventory estimate over the time cycle of the second period:

$$
\int_{0}^{T} I_{2}(t) d t=\frac{D}{\lambda+\theta}\left[\frac{\exp ((\lambda+\theta) T)-1}{\lambda+\theta}-T\right]=I_{2}^{T(\beta)}
$$

We now prove that inventory held in the second period in smaller with (higher) use of preservation technology (Lemma 4):

Proof: Similarly as the first period. The total inventory held during the second period with and without (lower) preservation is given by:

$$
\begin{gathered}
I_{2}{ }^{T(\beta)}=\int_{0}^{T} I_{2}(t)^{(\beta>0)} d t=\frac{D}{\lambda+\theta}\left[\frac{\exp ((\lambda+\theta) T)-1}{\lambda+\theta}-T\right], \\
I_{2}{ }^{T(0)}=\int_{0}^{T} I_{2}(t)^{(\beta=0)} d t=\frac{D}{\lambda+\alpha}\left[\frac{\exp ((\lambda+\alpha) T)-1}{\lambda+\alpha}-T\right]
\end{gathered}
$$

We estimate the term in the early bracket using a Taylor Series expansion:

$$
\int_{0}^{T} I_{2}(t)^{(\beta>0)} d t=\frac{D}{\lambda+\theta}\left[\frac{\left.(\lambda+\theta)+\frac{(\lambda+\theta)^{2}}{2}\right\}}{\lambda+\theta}-T\right],
$$




$$
\int_{0}^{T} I_{2}(t)^{(\beta=0)} d t=\frac{D}{\lambda+\alpha}\left[\frac{\left.(\lambda+\alpha)+\frac{(\lambda+\alpha)^{2}}{2}\right\}}{\lambda+\alpha}-T\right]
$$

Since:

$$
\operatorname{Exp}[(\gamma)(T)] \approx 1+[(\gamma)(T)]+\frac{[(\gamma)(T)]^{2}}{2}+\frac{[(\gamma)(T)]^{3}}{6}+\ldots
$$

The Lemma simplifies into proving:

$$
D\left[\frac{T^{2}}{2}+\frac{(\lambda+\theta) T^{3}}{6}\right]<D\left[\frac{T^{2}}{2}+\frac{(\lambda+\alpha) T^{3}}{6}\right]
$$

Which holds true because through Lemma $1(\alpha>\theta)$. $\square$

\section{Objective Function}

With all the important equations obtained, the objective function can be constructed from the Total Revenue $(T R)$ and Total Cost (TC).

\subsection{Total Revenue}

Total demand includes the small constant demand $(D)$ over the second period $(T)$ and the proportional demand $(\lambda)$ dependent on the inventory level held during both periods. Total revenue is thus the total demand multiplied by the selling price $(P)$ :

$$
T R=P D[T]+P \lambda \int_{0}^{t_{0}} I_{l}(t) d t+P \lambda \int_{0}^{T} I_{2}(t) d t
$$

\subsection{Total Cost}

Total cost includes Ordering cost $(O C)$, Holding cost $(H C)$, Preservation cost $(P C)$ and Deterioration cost $(D C)$. While ordering cost is just a multiple of the initial order quantity $(Q)$, the two other costs are inventory dependent and is a unit/time type of cost. Additionally they depend on the level of preservation employed $(\beta)$. Finally, Deterioration cost depends on the proportion of inventory that deterioration and only occurs during the second period.

$$
\begin{aligned}
T C & =C_{o} Q+\left[\frac{C_{h}}{\beta}+C_{p} \beta\right] \int_{0}^{t_{0}} I_{l}(t) d t \\
& +\left[\frac{C_{h}}{\beta}+C_{p} \beta+\frac{C_{d} \alpha}{1+\beta}\right] \int_{0}^{T} I_{2}(t) d t
\end{aligned}
$$

\subsection{Profit Function}

The profit function can thus be constructed using the total revenue and total cost functions. Total inventory held during period 1 and period 2 are common terms found in both functions allowing the simplification of the profit function:

$$
\begin{gathered}
\pi=T R-T C=[P D T]+\left[P \lambda-\frac{C_{h}}{\beta}-C_{p} \beta\right] \int_{0}^{t_{0}} I_{I}(t) d t \\
+\left[P \lambda-\frac{C_{h}}{\beta}-C_{p} \beta-\frac{C_{d} \alpha}{1+\beta}\right] \int_{0}^{T} I_{2}(t) d t-C_{o} Q
\end{gathered}
$$

The finalized objective function is the average total cycle profit $(A T C \pi)$ optimized by choosing the preservation factor $(\beta)$ and the second period cycle time $(T)$. Notice the first period cycle time $\left(t_{0}\right)$ would also depend on the two decision variables $(\beta)$ and $(T)$ through its expression:

$$
\max _{(\beta, T)}:[A T C \pi]=\pi / T^{T(\beta)}
$$

Where $\mathrm{T}^{\mathrm{T}(\beta)}=\mathrm{t}_{0}+\mathrm{T}=\left\{\frac{1}{\lambda} \ln \left[\frac{Q(\lambda+\theta)}{[\exp ((\lambda+\theta) T)-1] D}\right]\right\}+\mathrm{T}$

\subsection{Order Conditions}

Since the two decision variables $(\beta)$ and $(T)$ exists within multiple expressions in the objective function, it is easier to organize the order conditions with these expressions. The full expressions and their derivatives with respect to the two decision variables are provided in the appendix. The first and second order condition for the average profit function over the whole cycle time $\left(\frac{\pi}{t_{0}+T}\right)$ with $(\beta)$ and $(T)$ as decision variables are given as:

$$
\begin{aligned}
& \operatorname{ATC} \pi(\beta, T)=\frac{\pi}{T^{T(\beta)}}
\end{aligned}
$$

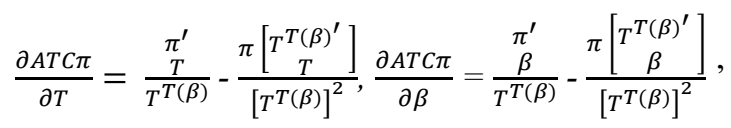

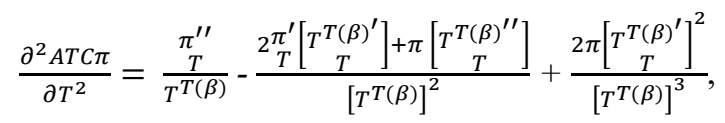

$$
\begin{aligned}
& \frac{\partial^{2} A T C \pi}{\partial \beta^{2}}=\frac{\pi^{\prime \prime}}{T^{T(\beta)}}-\frac{2^{\pi^{\prime}}\left[\begin{array}{c}
T^{T(\beta)^{\prime}} \\
\beta
\end{array}\right]+\pi\left[\begin{array}{c}
T^{T(\beta)^{\prime \prime}} \\
\beta
\end{array}\right]}{\left[T^{T(\beta)}\right]^{2}}+\frac{2 \pi\left[\begin{array}{c}
T^{T(\beta)^{\prime}} \\
\beta
\end{array}\right]^{2}}{\left[T^{T(\beta)}\right]^{3}}
\end{aligned}
$$

\subsection{Numerical Example}

To test out the model and find a possible solution, we use the following exogeneous parameters within the objective function to be maximized by finding the second period cycle time $\left(T^{*}\right)$ and preservation level $\left(\beta^{*}\right)$ :

$$
\begin{gathered}
Q=200000, D=35, \lambda=0.1, \alpha=0.2 \\
P=30, C_{d}=30, C_{0}=10, C_{h}=0.1, C_{p}=0.1
\end{gathered}
$$

\section{Solution Procedure}

We first specify the range for the two decision variables $(T)$ and $(\beta)$ that adhere to the assumptions of the model. The objective function is then proven to be strictly concave within the range of the two decision variables. The existence and uniqueness of a solution set $\left(\beta^{*}, T^{*}\right)$ is then proven using the strict concavity properties. An algorithm based on the generalized reduced gradient method to find the optimal solution is utilized as previously implemented $[20,25,26$, $37,39]$. Finally, the maximal nature of the solution is verified through the Hessian matrix as approached by previous research $[33,35,36]$.

\subsection{Range of Decision Variables}

The range of $(T)$ can be obtained from the explicit expression of $\left(\mathrm{t}_{0}\right)$ (lemma 5). To ensure deterioration kicks-in within the cycle time and not before the order is received, we need: 


$$
t_{0}>0 \text { or } \frac{1}{\lambda} \ln \left[\frac{Q(\lambda+\theta)}{[\exp ((\lambda+\theta) T)-1] D}\right]>0
$$

Which can only be observed if:

$$
\frac{Q(\lambda+\theta)}{[\exp ((\lambda+\theta) T)-1] D}>1 \text { or, } \frac{1}{\lambda+\theta} \ln \left[\frac{(\lambda+\theta) Q}{D}+1\right]>T
$$

Since:

$$
\log (\mathrm{x})>0 \text { when } \mathrm{x}>1 \text { for all } \mathrm{x} \in \mathrm{Q}^{+}
$$

This gives way to the upper bound for our decision variable $(\mathrm{T})$ :

$$
T \in\{0, \bar{T}\} \text { where, } \bar{T}=\frac{1}{\lambda+\theta} \ln \left[\frac{(\lambda+\theta) Q}{D}+1\right]
$$

For decision variable $(\beta)$, consider that we need a positive gain of inventory-proportional profit with the use of preservation: $\mathrm{K}=\left[\begin{array}{c}\pi^{\beta} \\ 2\end{array}\right]-\left[\begin{array}{c}\pi^{0} \\ 2\end{array}\right]>0$

$$
\mathrm{K}=\left[P \lambda-\frac{C_{h}}{\beta}-C_{p} \beta-\frac{C_{d} \alpha}{1+\beta}\right]-\left[P \lambda-C_{h^{-}} C_{d} \alpha\right]
$$

Since the preservation term $(\beta)$ is only found within $\left(\begin{array}{c}\pi^{\beta} \\ 2\end{array}\right)$, $\mathrm{K}$ is thus concave in $(\beta)$ :

$$
\frac{\partial K}{\partial \beta}=\frac{\partial^{\pi^{\beta}}}{\partial \beta}>0 \text { and } \frac{\partial^{2} K}{\partial \beta^{2}}=\frac{\partial^{2 \pi^{\beta}}}{\partial \beta^{2}}<0
$$

The maximum gain of inventory-proportional profit is thus at:

$$
\begin{gathered}
\frac{\partial^{2} K}{\partial \beta^{2}}=6 C_{p} \beta+2\left[C_{p}-C_{d} \alpha-C_{h}\right]=0 \text { or }, \\
\beta=\frac{\left[C_{d} \alpha+C_{h}-C_{p}\right]}{3 C_{p}}
\end{gathered}
$$

Beyond this point, additional investment in preservation is an over-investment since further increase in $(\beta)$ decrease inventory-proportional profit. Therefore preservation range is determined by inventory dependent cost and the natural deterioration rate (Lemma 6):

$$
\beta \in\{0, \bar{\beta}\} \text { where } \bar{\beta}=\frac{\left[C_{d} \alpha+C_{h}-C_{p}\right]}{3 C_{p}}
$$

\subsection{Concavity Properties}

(Theorem 1) The objective function is strictly concave within $T \in\{0, \bar{T}\}$.

Proof: We obtained the range of $(T)$ from Lemma 5 previously:

$T \in\{0, \bar{T}\}$ where,

$$
\bar{T}=\frac{1}{\lambda+\theta} \ln \left[\frac{(\lambda+\theta) Q}{D}+1\right] \text { and } t_{0}(\bar{T})=0
$$

Proving the statement below proves this theorem:

$$
\begin{gathered}
\delta A T C \pi(0)+(1-\delta) A T C \pi(\bar{T})< \\
A T C \pi[\delta(0)+(1-\delta)(\bar{T})] \text { for } \delta \in\{0,1\}
\end{gathered}
$$

Since $[A T C \pi(0)=0]$, the condition above simplifies into:

$$
A T C \pi(\bar{T})-(\delta) A T C \pi(\bar{T})<A T C \pi[(1-\delta)(\bar{T})]
$$

Consider the case when the value of $(\delta)$ is such that:

$$
\bar{T}=[(\delta) \bar{T}]+[(1-\delta) \bar{T}]=t_{0}+T
$$

We know that:

$$
A T C \pi[(1-\delta) \bar{T}]>A T C \pi(\bar{T})
$$

Since $A T C \pi[(1-\delta) \bar{T}]$ will involve a delay before deterioration (when deterioration starts taking effect with deterioration cost) and have a higher inventory-proportional profit over the total cycle time $(\bar{T})$ compared to $A T C \pi(\bar{T})$. This proves the above condition and the theorem. $\square$

(Theorem 2) The objective function is strictly concave within $\beta \in\{0, \bar{\beta}\}$.

Proof: We obtained the range of preservation from Lemma 6 previously:

$$
\beta \in\{0, \bar{\beta}\} \text { where } \bar{\beta}=\frac{\left[C_{d} \alpha+C_{h}-C_{p}\right]}{3 C_{p}}
$$

Proving Theorem 2 now simplifies into proving strict concavity of the objective function, which requires:

$$
\begin{gathered}
A T C \pi[\delta(0)+(1-\delta)(\bar{\beta})]> \\
\delta A T C \pi(0)+(1-\delta) A T C \pi(\bar{\beta}) \text { for } \delta \in\{0,1\}
\end{gathered}
$$

Or alternatively:

$$
A T C \pi[(1-\delta)(\bar{\beta})]>\delta A T C \pi(0)+(1-\delta) A T C \pi(\bar{\beta})
$$

We may assume the optimal value of $\left(T^{*}\right)$ since the range of $(\beta)$ is independent if $(T)$. The condition above can be proven using the set of exogeneous variables in the numerical example to firstly determine $(\bar{\beta})$ before calculating the value of the maximized objective function $(A T C \pi)$ for each level of $(\beta)$. $\square$

(Theorem 3) There exists a unique optimal solution set for the objective function.

Proof: Both $\left(T^{*}\right)$ and $\left(\beta^{*}\right)$ lie within their respective closed sets. Since the objective function is also continuous on the bounded sets, there exists at least one maximum point for the objective function. Through Theorem 1 and Theorem 2, we proved that the objective function is strictly concave within the range of our 2 decision variables. Therefore a maximum point obtained by the solution set $\left(\beta^{*}, T^{*}\right)$ must be a global maximum since any strictly concave function will have at most one maximal point.

\subsection{Algorithm}

1) Set $\left(\beta_{n}=C_{h}\right)$ and $\left(T_{n}=1\right)$ with $(n=0)$.

2) If $\left(\frac{\partial A T C \pi}{\partial \beta}\right)$ and $\left(\frac{\partial A T C \pi}{\partial T}\right)$ are both negative, go to step 8 .

3) If $\left(\frac{\partial A T C \pi}{\partial \beta}<0\right)$, skip to step 5 .

4) Solve for optimal $(\beta)$, If $(\triangle A T C \pi>0.1)$, update $\left(\beta_{n}\right)$ to the newly solve value. 
5) If $\left(\frac{\partial A T C \pi}{\partial T}<0\right)$, skip to step 7 .

6) Solve for optimal $(T)$, if $(\triangle A T C \pi>0.1)$, update $\left(T_{n}\right)$ to the newly solved value.

7) If $\left|\beta_{n}-\beta_{n-1}\right|+\left|T_{n}-T_{n-1}\right|>0.001$ go to step 3. If not, go step 8.

8) STOP. The last updated value is an optimal solution set $\left(\beta^{*}, T^{*}\right)$.

We find the optimal cycle time $\left(T^{*}=20.961\right)$ and optimal level of preservation level $\left(\beta^{*}=0.65754\right)$ through the Algorithm with an Average Total Cycle Profit $(A T C \pi)$ of (64964.58).

To establish the maximal nature of our obtained solution, we verify the principal minor and major of the Hessian matrix evaluated at the solution set $\left(\beta^{*}, T^{*}\right)$. The negative value for the principal minor (concavity for either decision variable) was proven through Theorem 1 and 2 . We can check the positive definite sign for the principal major. This reaffirms the maximum point obtained from our solution:

$$
\left|H_{2}\right|=\left[\frac{\partial^{2} A T C \pi}{\partial \beta^{2}}\right]^{*}\left[\frac{\partial^{2} A T C \pi}{\partial T^{2}}\right]-\left[\frac{\partial^{2} A T C \pi}{\partial \beta \partial T}\right]>0
$$

The graphical representation for the objective function against the two decision variable, the level of Preservation $(\beta)$ and second period cycle time $(T)$ is given below:

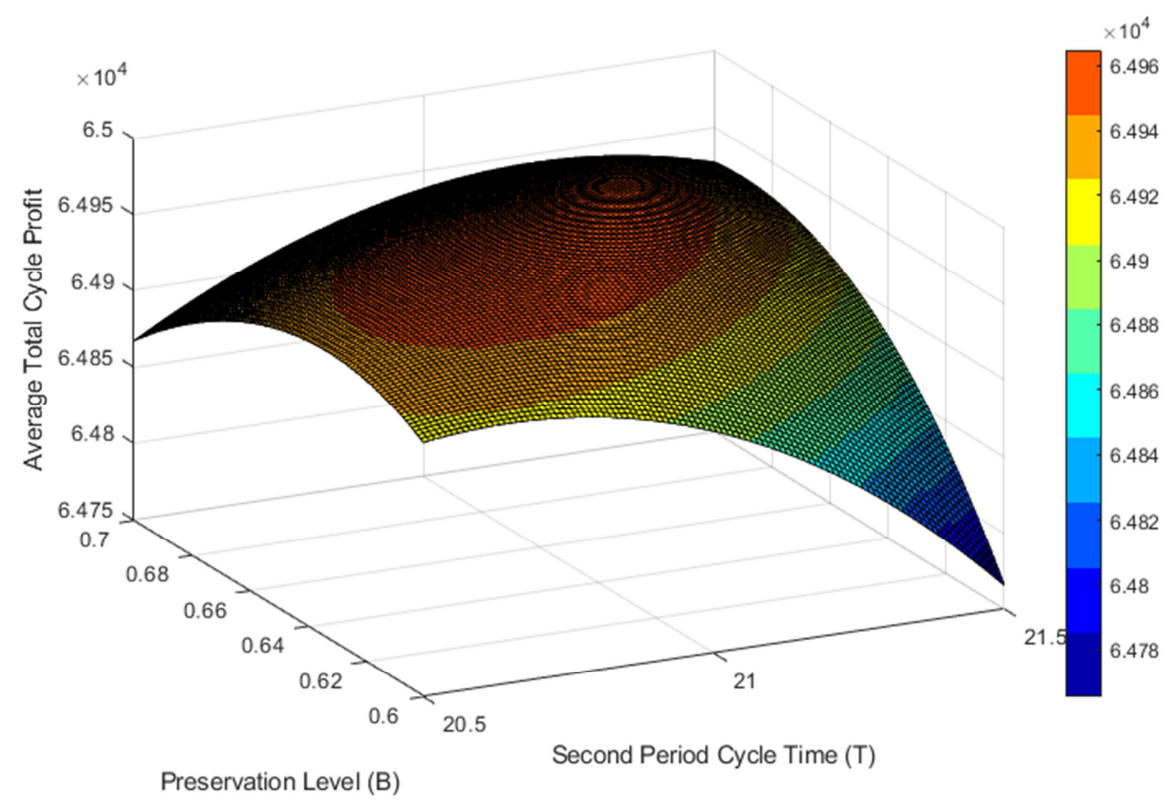

Figure 2. ATC $\pi$ (y-axis) with variation in Total Cycle Time (T) (z-axis) and Preservation Level $(\beta)(x$-axis).

\subsection{Gains from Using Preservation Technology}

While it is clear to see the optimal solutions give the maximum point through the graph, the channels by which preservation increases profit is still uncertain. Since there is evident gains (and possible loss) that could come from the level of preservation, further investigation is needed to actually understand how preservation technology can affect profits. The difference in Average Total Cycle Profit between businesses that uses preservation $\left(A T C \pi^{(\beta)}\right)$ and a model that does not $\left(A T C \pi^{(0)}\right)$ can be organized into each of their related exogenous terms. We look into all 5 terms separately:

$$
\begin{gathered}
A T C \pi^{(\beta)}-A T C \pi^{(0)}=A+B+C+D+E \\
A=[P \lambda] *\left[\frac{I_{1}^{T(\beta)}+I_{2}{ }^{T(\beta)}}{T^{T(\beta)}}-\frac{I_{1}{ }^{T(0)}+I_{2}{ }^{T(0)}}{T^{T(0)}}\right]
\end{gathered}
$$

For A, the difference between average inventory held for the model with and without preservation determines how much gain it contributes. Through Lemma 3 it is proven that inventory held during the first period increase with higher levels of preservation. As a balance, inventory held during second period decreases through Lemma 4. Nonetheless, notice that total cycle time increases with higher usage of preservation according to Lemma 2. Therefore overinvestment in preservation may eliminate this gain.

$$
B=\left[-C_{h}\right] *\left[\frac{I_{1}^{T(\beta)}+I_{2}{ }^{T(\beta)}}{\beta T^{T(\beta)}}-\frac{I_{1}{ }^{T(0)}+I_{2}{ }^{T(0)}}{T^{T(0)}}\right]
$$

For B, the term in the right bracket is similar as before, this time greater inventory held would entail greater total holding cost. Additionally the inventory dependent per unit time holding cost with preservation is discounted proportionally, reducing total holding cost when preservation is used.

$$
C=\left[-C_{p} \beta\right] *\left[\frac{I^{I_{1}(\beta)}+I_{2}{ }^{T(\beta)}}{T^{T(\beta)}}\right]
$$

For $\mathrm{C}$, the preservation cost which is only applicable when preservation is employed increases firstly according to the level of utilization in the effective preservation cost in the left bracket. Secondly the preservation cost is incurred through the average inventory held during the whole cycle.

$$
D=\left[C_{d}\right] *\left[\frac{I_{2}{ }^{T(0)} \alpha}{T^{T(0)}}-\frac{I_{2}{ }^{T(\beta)} \alpha}{T^{T(\beta)}}\right]
$$

Next, term $\mathrm{D}$ is the difference in deterioration cost incurred when preservation is utilized. This is where the 
majority of cost is saved in deteriorating inventory models with preservation technologies. Through Lemma 4, it can be seen that average inventory held during the second period is smaller with the use of preservation. Additionally, the effective deterioration rate is reduced through Lemma 1.

$$
E=\left[C_{0} Q\right] *\left[\frac{1}{T^{T(0)}}-\frac{1}{T^{T(\beta)}}\right]
$$

Finally, the last term is the difference in spread of the initial ordering quantity cost based on Lemma 2. Since the length of first period is greater with preservation, the initial ordering cost is spread over a longer period with higher preservation levels.

The gains in $(A T C \pi)$ occur through the alteration of inventory process, which prolongs the delay before deterioration (first period cycle time) and reduces the effective deterioration $\theta<\alpha$ (Preposition 1). By prolonging the delay before preservation, the total costs are spread further as most evidently seen in term E. Meanwhile by reducing the effective deterioration rate, the amount of inventory that is lost to deterioration is reduced as seen in term D. Additionally, the amount of inventory held in the first period is increased while the amount of inventory held in the second period is reduced as proven through Lemmas 3 and 4. As a whole, this shifts the weight of the operation cycle towards the first period where no deterioration cost is incurred.

The gains in $(A T C \pi)$ affected by the changes in inventoryassociated costs namely the additional preservation cost $\left(C_{p} \beta\right)$ and the savings of holding cost due to utilization of preservation $\left(\frac{C_{h}}{\beta}\right)$ (Preposition 2 ). We can see this effect clearly within term $\mathrm{C}$ where successive increments in preservation will quickly increase through the per unit preservation cost in the left bracket. On the contrary in term $\mathrm{B}$, successive increments in preservation has a positive effect by improving inventory durability that reduces per unit holding cost.

\section{Sensitivity Analysis}

We conduct sensitivity analysis to verify Prepositions 1 and 2. The tables below show the differences in Average Total Cycle Profit with differing levels of Total Cycle Time and Preservation Level when exogenous costs change.

Consider an increase in deterioration cost from 30 to 34 and subsequently 38. From term D previously, we found in Preposition 1 that it would be beneficial to increase preservation levels higher to reduce the amount of inventory susceptible to deterioration in the second period. The more expensive deterioration cost, the more gains that could come from increasing preservation levels as seen in tables 2 and 3:

Table 3. ATC $\pi$ values for $C_{d}=34$.

\begin{tabular}{llllll}
\hline $\boldsymbol{\beta} / \mathbf{T}$ & $\mathbf{1 9}$ & $\mathbf{2 0}$ & $\mathbf{2 0 . 9 6 1}$ & $\mathbf{2 1}$ & $\mathbf{2 2}$ \\
\hline 0.55 & 64584 & 64757 & 64577 & 64527 & 63755 \\
0.6 & 64624 & 64878 & 64812 & 64780 & 64211 \\
0.65 & 64566 & 64889 & 64923 & 64907 & 64518 \\
0.7 & 64464 & 64830 & 64927 & 64921 & 64649 \\
0.75 & 64305 & 64709 & 64865 & 64868 & 64704 \\
\hline
\end{tabular}

Table 4. ATC $\pi$ values for $C_{d}=38$.

\begin{tabular}{llllll}
\hline $\boldsymbol{\beta} / \mathbf{T}$ & $\mathbf{1 9}$ & $\mathbf{2 0}$ & $\mathbf{2 0 . 9 6 1}$ & $\mathbf{2 1}$ & $\mathbf{2 2}$ \\
\hline 0.55 & 64234 & 64299 & 63997 & 63930 & 62976 \\
0.6 & 64304 & 64461 & 64286 & 64239 & 63509 \\
0.65 & 64275 & 64511 & 64448 & 64418 & 63888 \\
0.7 & 64192 & 64479 & 64488 & 64469 & 64068 \\
0.75 & 64053 & 64385 & 64460 & 64452 & 64172 \\
\hline
\end{tabular}

We now consider changes in Preservation Cost from 0.1 to 0.3 and subsequently 0.5 . From term $\mathrm{C}$ of section 5.8 , it is discussed through Preposition 2 that it will be more expensive to employ higher preservation levels, making it less beneficial to have high levels of preservation. Observing tables 4 and 5 below confirm predictions from Preposition 2:

Table 5. ATC $\pi$ values for $C_{p}=34$.

\begin{tabular}{llllll}
\hline $\boldsymbol{\beta} / \mathbf{T}$ & $\mathbf{1 9}$ & $\mathbf{2 0}$ & $\mathbf{2 0 . 9 6 1}$ & $\mathbf{2 1}$ & $\mathbf{2 2}$ \\
\hline 0.55 & 60706 & 60901 & 60770 & 60729 & 60061 \\
0.6 & 60378 & 60638 & 60604 & 60578 & 60089 \\
0.65 & 59919 & 60234 & 60283 & 60271 & 59937 \\
0.7 & 59503 & 59851 & 59952 & 59948 & 59715 \\
0.75 & 58997 & 59372 & 59520 & 59524 & 59383 \\
\hline
\end{tabular}

Table 6. ATC $\pi$ values for $C_{p}=38$.

\begin{tabular}{llllll}
\hline $\boldsymbol{\beta} / \mathbf{T}$ & $\mathbf{1 9}$ & $\mathbf{2 0}$ & $\mathbf{2 0 . 9 6 1}$ & $\mathbf{2 1}$ & $\mathbf{2 2}$ \\
\hline 0.55 & 56477 & 56587 & 56384 & 56333 & 55589 \\
0.6 & 55810 & 55982 & 55871 & 55835 & 55263 \\
0.65 & 54980 & 55201 & 55169 & 55147 & 54726 \\
0.7 & 54271 & 54521 & 54538 & 54524 & 54199 \\
0.75 & 53436 & 53710 & 53771 & 53764 & 53528 \\
\hline
\end{tabular}

We graph the data to show optimal level of preservations does follow the notions of prepositions 1 and 2 . Increasing deterioration costs entails greater optimal preservation level while increasing preservation costs will require lower levels of optimal preservation:
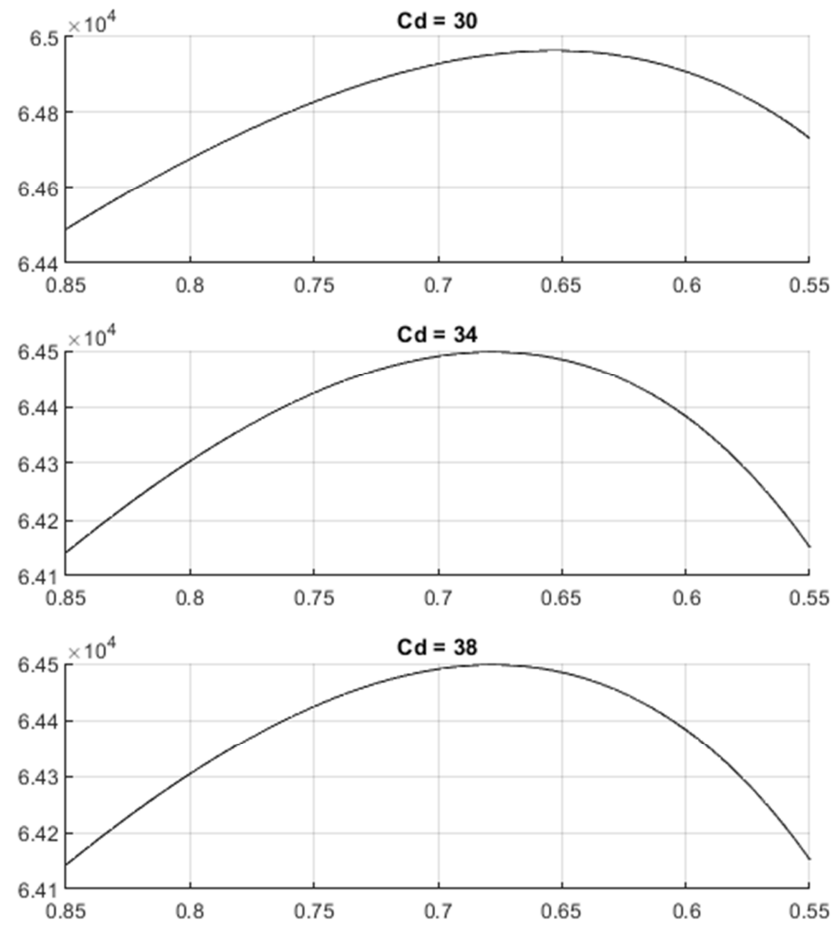

Figure 3. Optimal Preservation Levels $(\beta)$ with increase in Deterioration $\operatorname{Cost}\left(C_{d}\right)$. 

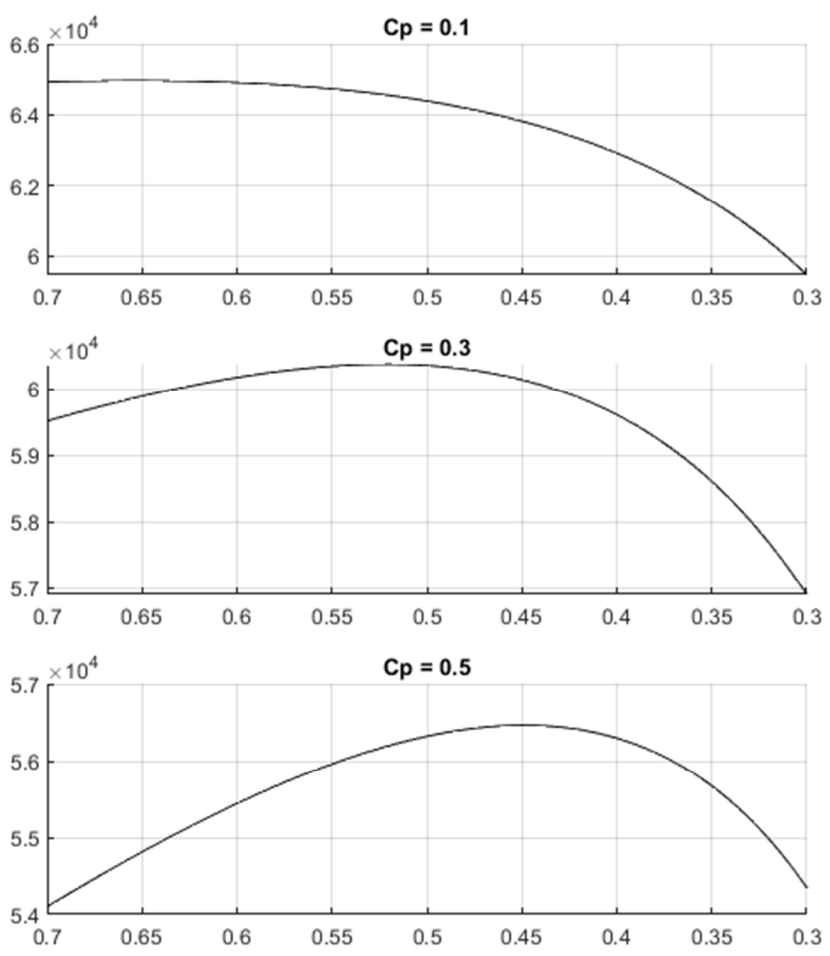

Figure 4. Optimal Preservation Levels $(\beta)$ with increase in Preservation $\operatorname{Cost}\left(C_{p}\right)$.

Gains in employing additional levels of preservation would depend on the returns to scale of the preservation technology towards inventory related cost and effective deterioration rate. (Preposition 3)

Consider the additional dimension of inventory-associated cost where there are differing returns to scale. Increasing levels of preservation could require increasing increments to the preservation cost. Now increase in preservation levels increases preservation cost at an increasing rate. This would limit the gain from preservation, lowering the optimal level of preservation. This is more clearly seen where term $\mathrm{C}$ changes into:

$$
C=\left[-C_{p} \beta^{n}\right]\left[\frac{I_{1}{ }^{T(\beta)}+I_{2}{ }^{T(\beta)}}{T^{T(\beta)}}\right] \text { where }(n>1)
$$

Equally interesting would be decreasing scale to holding cost savings. Now there would be less difference in total holding cost when preservation is employed since higher level of preservation would not continuously reduce holding cost. Similar as before, this would lower the optimal level of preservation. This can be seen where term $B$ changes into:

$$
B=\left[-C_{h}\right]\left[\frac{I_{1}{ }^{T(\beta)}+I_{2}{ }^{T(\beta)}}{\left(\beta^{m}\right) T^{T(\beta)}}-\frac{I_{1}{ }^{T(0)}+I_{2}{ }^{T(0)}}{T^{T(0)}}\right]
$$

where, $(m<1)$

The final consideration is varying effectiveness for preservation in reducing effective deterioration as seen below:

$$
\theta(\mathrm{k})=\frac{\alpha}{1+\left(\beta^{k}\right)}, \mathrm{I}_{2}(\mathrm{t})=\frac{D}{\lambda+\theta k}[\exp ((\lambda+\theta(k))(\mathrm{T}-\mathrm{t}))-1]
$$

This would alter the initial condition that implicitly determines first period cycle time $\left(t_{0}\right)$ and total cycle time. The value of $(k)$ would also determine the extent of delay that would be incurred via the preservation investment. Values of $(k>1)$ would signal increasing effectiveness of preservation investment in reducing effective deterioration. The same amount of decrease in effective deterioration rate would require a lower level of preservation as opposed to an ineffective preservation situation with $(k<1)$ values. Ultimately this will affect total inventory held during both periods, a major component in the objective function and affecting all 5 terms relating to the gains from preservation.

\section{Future Research}

Possible extensions and improvements for this study may consider further detailed effects of preservation technologies in its effectiveness and the salvage value for deteriorated items that have been preserved.

As implied through Preposition 3, differing returns to scale and increments in preservation costs could further explain until which exact point preservation continues to give benefit to the business. The benefits to employing preservation technology may then be attributed to each specific cost or revenue component rather than just the overall profit. Such an approach has not been taken since previous papers consider linearly increasing preservation costs even with diminishing returns to scale for preservation.

Finally, there is no salvage value consideration and discussion. Since inventories are well kept and preserved during the whole cycle, deteriorated items could be sold off at a discount once they deteriorate $[22,36,40]$. There could be cost recuperated from this avenue that should be explored.

\section{Conclusion}

In this paper, it is demonstrated that the average total cycle profit could be maximized by choosing the optimal level of preservation $\left(\beta^{*}\right)$ and second period cycle time $\left(T^{*}\right)$ in a non-instantaneous deteriorating inventory setting. We discover two channels by which preservation increases profit, namely by altering the inventory process and the changes in inventory costs affected by the level of preservation utilized.

Through the alteration of inventory process, we find that a higher level of preservation prolongs the delay before deterioration and increases the inventory held during the first period. This essentially shifts weight and majority of the operation cycle to the higher profitable first period in line with Preposition 1. The derivation on how the level of preservation determines the time when inventory process changes is explicitly demonstrated in this research, improving the current limited literature on this issue.

Additionally, it is found with Preposition 2 that businesses stand to gain (or lose) depending on the inventory dependent costs incurred during the cycle. Favorably, preservation cost is now incurred more accurately according to the level of inventory held. Holding costs during the cycle are also reduced owing to more durable inventory requiring less 
delicate care. While it is beneficial to employ sufficiently high levels of preservation, successively increasing preservation levels might require increasing increments to the preservation cost as suggested through Preposition 3.

Industries and businesses with easily-perishable high value products like caviar and radioactive materials would greatly benefit from the findings of this research. There are great savings that would arise from the reduced deterioration cost and inventory preserved to satisfy demand when the appropriate operation cycle time and level of preservation are chosen. Future research in this area could consider varying preservation technologies effectiveness that have diminishing or increasing increments towards inventory related costs.

\section{Acknowledgements}

This work was supported by the Frontier Research Grant under University Malaya entitled, Advanced Mathematical Modelling For Warehouse Operation (FG039-17AFR).

\section{Appendix: Order Conditions}

A) Order conditions for per inventory proportional profit in period 1 and 2:

$$
\begin{gathered}
\pi_{l}(\beta)=\left[P \lambda-\frac{C_{h}}{\beta}-C_{p} \beta\right] \pi_{2}(\beta)=\left[P \lambda-\frac{C_{h}}{\beta}-C_{p} \beta-\frac{C_{d} \alpha}{1+\beta}\right] \\
\pi_{1 \beta}{ }^{\prime}=\left[\frac{C_{h}}{\beta^{2}}-C_{p]} \pi_{2 \beta}{ }^{\prime}=\left[\frac{C_{h}}{\beta^{2}}-C_{p}-\frac{C_{d} \alpha}{(1+\beta)^{2}}\right]\right. \\
\pi_{1 \beta}{ }^{\prime \prime}=\left[\frac{-2 C_{h}}{\beta^{3}}-C_{p]} \pi_{2 \beta}{ }^{\prime \prime}=-2\left[\frac{C_{h}}{\beta^{3}}+\frac{C_{d} \alpha}{(1+\beta)^{3}}\right]\right.
\end{gathered}
$$

B) Order conditions for Inventory held in period 1:

$$
\begin{gathered}
I_{I}^{T(\beta)}(\beta, T)=\int_{0}^{t 0} I_{I}(t) d t=\frac{Q}{\lambda}\left[1-\frac{1}{\exp (\lambda t 0)}\right] \\
I_{I T}{ }^{T(\beta)^{\prime}}=\frac{Q(\lambda+\theta) \exp ((\lambda+\theta) T)}{[\lambda \exp (\lambda t 0)][1-\exp ((\lambda+\theta) T)]} \\
I_{I T}^{T(\beta)^{\prime \prime}}=\frac{Q(\lambda+\theta) \exp ((\lambda+\theta) T)}{[\lambda \exp (\lambda t 0)][\exp ((\lambda+\theta) T)-1]} \\
I_{l \beta}^{T(\beta)^{\prime}}=\frac{Q \alpha \lambda}{[(1+\beta) \lambda \exp (\lambda t 0)]^{2}}\left\{\frac{(T) \exp ((\lambda+\theta) T)}{[\exp ((\lambda+\theta) T)-1]}-(\lambda+\theta)\right\} \\
I_{l \beta}^{T(\beta)^{\prime \prime}}=\frac{Q \alpha^{3} \lambda}{\left[(1+\beta)^{4}[\lambda \exp (\lambda t 0)]^{2}\right.}\left\{\frac{T^{2} \exp ((\lambda+\theta) T)}{[\exp ((\lambda+\theta) T)-1]}+1\right\} \\
+\left\{\frac{Q \alpha \lambda^{3} \exp (\lambda t 0)}{\left[(1+\beta)^{4}[\lambda \exp (\lambda t 0)]^{4}\right.}\left[\frac{T \exp ((\lambda+\theta) T)}{[\exp p((\lambda+\theta) T)-1]}-(\lambda+\theta)\right]\right\} \\
\left\{\left[(\lambda+\theta)-\frac{T \exp ((\lambda+\theta) T)}{[\exp p((\lambda+\theta) T)-1]}\right]-[\lambda \exp ((\lambda+\theta) T)]\right\}
\end{gathered}
$$

C) Order conditions for Inventory held in period 2:

$$
\begin{gathered}
I_{2}^{T(\beta)}(\beta, T)=\int_{0}^{T} I_{2}(t) d t=\frac{D}{\lambda+\theta}\left[\frac{\exp ((\lambda+\theta) T)-1}{\lambda+\theta}-T\right] \\
I_{2 T^{T}}{ }^{T(\beta)^{\prime}}=\frac{D[\exp ((\lambda+\theta) T)-1]}{\lambda+\theta}, \quad I_{2 T}{ }^{T(\beta) ”}=D[\exp ((\lambda+\theta) T)] \\
I_{2 \beta}{ }^{T(\beta)^{\prime}}=\frac{\alpha D}{(\lambda+\theta)^{2}(1+\beta)^{2}}\{(\lambda+\theta) \exp ((\lambda+\theta) T)[2-((\lambda+\theta) T)]-2(\lambda+\theta)-T\} \\
I_{2 \beta}{ }^{T(\beta)^{\prime \prime}=}\left\{\frac{2[\alpha D][\lambda+\theta][\alpha-(\lambda+\theta)(1+\beta)]}{(\lambda+\theta)^{4}(1+\beta)^{4}}\right\}\{(\lambda+\theta) \exp ((\lambda+\theta) T)[2-((\lambda+\theta) T)]-2(\lambda+\theta)-T\}+ \\
\left\{\frac{\alpha D}{(\lambda+\theta)^{2}(1+\beta)^{4}}\right\}\{2(\lambda+\theta) \alpha+[T(\lambda+\theta) \exp ((\lambda+\theta) T)][T(\lambda+\theta)+2]-2 \alpha \exp ((\lambda+\theta) T)[(\lambda+\theta)+1]
\end{gathered}
$$

D) Order conditions for end of period 1:

$$
\begin{gathered}
t_{0}=\frac{1}{\lambda} \ln \left[\frac{Q(\lambda+\theta)}{[\exp ((\lambda+\theta) T)-1] D}\right] \\
t_{0 \beta}=\frac{\alpha}{\lambda(\lambda+\theta)(1+\beta)^{2}}\left[\frac{T \exp ((\lambda+\theta) T)(\lambda+\theta)}{[\exp ((\lambda+\theta) T)-1]}-1\right]
\end{gathered}
$$




$$
\begin{gathered}
t_{0 T}{ }^{\prime}=\frac{\exp ((\lambda+\theta) T)(\lambda+\theta)}{\lambda[1-\exp ((\lambda+\theta) T)]} \\
t_{0 \beta}{ }^{\prime \prime}=\frac{\alpha^{2}}{\lambda(\lambda+\theta)(1+\beta)^{4}}\left[\frac{T^{2} \exp ((\lambda+\theta) T)}{[\exp ((\lambda+\theta) T)-1]}-\frac{1}{(\lambda+\theta)^{2}}\right]+\frac{\alpha[\alpha-2(\lambda+\theta)]}{\lambda(\lambda+\theta)^{2}(1+\beta)^{3}}\left[\frac{T^{2} \exp ((\lambda+\theta) T)}{[\exp ((\lambda+\theta) T)-1]}-\frac{1}{(\lambda+\theta)}\right] \\
t_{0 T}{ }^{\prime}=\frac{\alpha[1-\exp ((\lambda+\theta) T)]^{2} \exp ((\lambda+\theta) T)}{(1+\beta)^{2}}\{((\lambda+\theta) T)-1-(\lambda+\theta) T\}
\end{gathered}
$$

E) Order conditions for total cycle time:

$$
\begin{aligned}
& T^{T(\beta)}=t_{0}+T \\
& T_{T}^{T(\beta){ }^{\prime}}=t_{0 T}{ }^{\prime}+1 \quad T_{T}^{T(\beta) "}=t_{0 T}{ }^{\prime} \\
& T_{\beta}{ }^{T(\beta)}{ }^{\prime}=t_{0 \beta}{ }^{\prime} \quad T_{\beta}{ }^{T(\beta)}{ }^{\prime}=t_{0 \beta}{ }^{\prime}
\end{aligned}
$$

F) Order conditions for total profit:

$$
\begin{aligned}
& \pi=[P D T]-\left[C_{o} Q\right]+\left[\pi_{1} I_{1}^{T(\beta)}\right]+\left[\pi_{2} I_{2}^{T(\beta)}\right] \\
& \pi_{T}^{\prime}=[P D]+\left[\pi_{1} I_{l T}^{T(\beta)^{\prime}}\right]+\left[\pi_{2} I_{2 T}^{T(\beta)^{\prime}}\right] \\
& \pi_{T}{ }^{\prime \prime}=\left[\pi_{1} I_{1 T}^{T(\beta) "}\right]+\left[\pi_{2}{ }_{I 2 T}^{T(\beta) "}\right] \\
& \text { Since } \pi_{1 T}{ }^{\prime}=0, \pi_{1 T}{ }^{\prime}=0, \pi_{2 T}{ }^{\prime}=0, \pi_{2 T}{ }^{\prime}=0 \\
& \pi=[P D T]-\left[C_{o} Q\right]+\left[\pi_{1} I_{1}^{T(\beta)}\right]+\left[\pi_{2} I_{2}^{T(\beta)}\right] \\
& \pi_{\beta}{ }^{\prime}=\left(\pi_{1 \beta}{ }^{\prime} I_{1}^{T(\beta)}+\pi_{1} I_{1 \beta}{ }^{T(\beta)^{\prime}}\right)+\left(\pi_{2 \beta}{ }^{\prime} I_{2}^{T(\beta)}+\pi_{2} I_{2 \beta}{ }^{T(\beta)^{\prime}}\right) \\
& \left.\pi_{\beta}{ }^{\prime}=\left[\left(\pi_{1 \beta}{ }^{\prime \prime} I_{l}^{T(\beta)}+\pi_{1 \beta}{ }^{\prime} I_{1 \beta}{ }^{T(\beta)^{\prime}}\right)+\left(\pi_{1 \beta}{ }^{\prime} I_{1 \beta}{ }^{T(\beta)^{\prime}}+\pi_{1} I_{1 \beta}{ }^{T(\beta)}{ }^{\prime \prime}\right)\right]+\left[\left(\pi_{2 \beta}{ }^{\prime \prime} I_{2}^{T(\beta)}+\pi_{2 \beta}{ }^{\prime} I_{2 \beta}{ }^{T(\beta)^{\prime}}\right)+\left(\pi_{2 \beta}{ }^{\prime} I_{2 \beta}{ }^{T(\beta)^{\prime}}+\pi_{2} I_{2 \beta}{ }^{T(\beta)}\right)^{\prime \prime}\right)\right]
\end{aligned}
$$

\section{References}

[1] A. A. Taleizadeh, An economic order quantity model for a deteriorating item in a purchasing system with multiple prepayments. Applied Mathematical Modelling (2014).

[2] A. K Bhunia, C. K Jaggi, A. Sharma and R. Sharma, A twowarehouse inventory model for deteriorating items under permissible delay in payment with partial backlogging. Applied Mathematics and Computation (2014).

[3] A. K Bhunia and A. A Shaikh, An application of PSO in a two-warehouse inventory model for deteriorating item under permissible delay in payment with different inventory policies. Applied Mathematics and Computation (2015).

[4] B. Sarkar and S. Sarkar, An Improved Inventory Model with Partial Backlogging, Time Varying Deterioration and StockDependent Demand, Economic Modelling, 30 (2013), 924932.

[5] B. Sarkar, A Production-Inventory Model with Probablilistic Deterioration in Two-Echelon Supply Chain Management, Applied Mathematical Modelling, 37 (2013), 3138-3151.

[6] B. Sarkar and S. Sarkar, Variable Deterioration and DemandAn Inventory Model, Economic Modelling, 31 (2013), 548556.

[7] B. C. Giri and K. S. Chaudhuri, Deterministic Models of Perishable Inventory With Stock-Dependent Demand Rate and Nonlinear Holding Cost, European Journal of Operational Research, 105 (1998), 467-474.

[8] B. C. Giri, A. Goswami and K. S. Chaudhuri, An EOQ
Model for Deteriorating Items with Time Varying Demand and Costs. Journal of the Operational Research Society, 47 (1996), 1398.

[9] C. K. Chan, W. H. Wong, A. Langevin and Y. C. E. Lee, An integrated production-inventory model for deteriorating items with consideration of optimal production rate and deterioration during delivery. International Journal of Production Economics, 189 (2017), 1, 1-13.

[10] C. T Yang, C. Y Dye and J. F Ding, Optimal dynamic trade credit and preservation technology allocation for a deteriorating inventory model, Computers \& Industrial Engineering, 87, September (2015), 356-369.

[11] C. Y Dye, The effect of preservation technology investment on a non-instantaneous deteriorating inventory model, Omega, Volume 41 (5) (2013), 872-880.

[12] C. Y Dye, C. T Yang and C. C Wu, Joint dynamic pricing and preservation technology investment for an integrated supply chain with reference price effects, Journal of the Operational Research Society, 69 (2017), Issue 6, 811-824.

[13] C. Y. Dye, The effect of preservation technology investment on a non-instantaneous deteriorating inventory model. Omega 41 (2013), 5, 872.

[14] G. Li, X. He, J. Zhou and H. Wu, Pricing, Replenishment and Preservation Technology Investment Decision for NonInstantaneous Deteriorating Items, Omega, 84 (2019), 114126.

[15] H. Huang, Y. He and D. li, Pricing and Inventory Decisions in the Food Supply Chain with Production Disruption and Controllable Deterioration, Journal of Cleaner Production, 180 (2018), 280-296. 
[16] H. K. Alfares, Inventory model with stock-level dependent demand rate and variable holding cost. International Journal of Production Economics, 108 (2007), 259-265.

[17] J. T. Teng, M. S. Chern, H. L. Yang and Y. J. Wang, Deterministic lot-size Inventory Models With Shortages and Deterioration for Fluctuating Demand, Operations Research Letters, 24 (1999), 65-72.

[18] J. Zhang, Q. Wei, Q. Zhang and W. Tang, Pricing, Service and Preservation Technology Investment Policy for Deteriorating Items Under Common Resource Constraints, Computers and Industrial Engineering, 95 (2016), 1-9.

[19] J. T. Teng, L. Eduardo C. Barron, H. J. Chang, J. Wu and Y. $\mathrm{Hu}$, Inventory Lot-Sizes for Deteriorating Items With Expiration Dates and Advance Payments, Applied Mathematical Modelling, 40 (2016), 8605-8616.

[20] K-J Chung, J-J Liao, P-S Ting, S-D Lin and H. M Srivastava, The algorithm for the optimal cycle time and pricing decisions for an integrated inventory system with order-size dependent trade credit in supply chain management. Applied Mathematics and Computation (2015).

[21] L. Liu, L. Zhao and X. Ren, Optimal preservation technology investment and pricing policy for fresh food, Computers \& Industrial Engineering, 135, September (2019), 746-756.

[22] M. Rabbani, N. Pourmohammad and H. Rafiei, Joint optimal dynamic pricing and replenishment policies for items with simultaneous quality and physical quantity. Applied Mathematics and Computation (2016).

[23] P. M. Ghare, G. F. Schrader, A Model for an Exponential Decaying Inventory. Journal of Industrial Engineering, 14 (1963), 283-243.

[24] P. H. Hsu, H. M. Wee, H. M. Teng, Preservation technology investment for deteriorating inventory, International Journal of Production Economics, 124 (2010), 2, 388.

[25] R. Maihami and I. N. K. Abadi, Joint Control of Inventory and Its Pricing for Non-Instantaneously Deteriorating Items Under Permissible delay in Payments and Partial Backlogging, Mathematical and Computer Modelling, 55 (2012), 1722-1733.

[26] R. Maihami and B. Karimi, Optimizing the Pricing and Replenishment Policy for Non-Instantaneous Deteriorating Items with Stochastic Demand and Promotional Efforts, Computers \& Operations Research, 51 (2014), 302-312.

[27] R. Covert and G. C. Philip, An EOQ Model for Items with Weibull Distribution. AIIE Transactions, 5 (1973), 323-326.

[28] S. R. Singh and S. Sharma, A Global Optimizing Policy for Decaying Items with Ramp-Type Demand Rate under TwoLevel Trade Credit Financing Taking Account of Preservation Technology, Advances in Decision Sciences, (2013), Article ID 126385 .
[29] S. R. Singh, S. Jain and H. Dem, Two Storage Production Model With Imperfect Quality For Decaying Items Under Preservation, International Conference on Computational Intelligence: Modelling, Techniques and Applications, Procedia Technology 10 (2013), 208-215.

[30] S. Saha, I. Nielsen and I. Moon, Optimal Retailer Investments in Green Operations and Preservation Technology for Deteriorating Items, Journal of Cleaner Production, 140 (2017), 1514-1527.

[31] S. Pal, A. Goswami and K. S. Chaudhuri, A deterministic inventory model for deteriorating items with stock-dependent demand rate. International Journal of Production Economics, 32 (1993), 291-299.

[32] T. P. Hsieh and C. Y. Dye, Optimal dynamic pricing for deteriorating items with reference price effects when inventories stimulate demand, European Journal of Operational Research, 262 (2017), Issue 1, 136-150.

[33] T. Singh, P. J. Mishra and H. Pattanayak, An Optimal Policy for Deteriorating Items with time-proportional Deterioration Rate and Constant and Time-Dependent Linear Demand Rate, Journal of Industrial Engineering, 13 (2017), 455-463.

[34] U. Mishra, J. Z. Wu, Y. C. Tsao and M. L. Tseng, Sustainable Inventory System with Controllable Non-Instantaneous Deterioration and Environment Emission Rates, Journal of Cleaner Production (2019).

[35] V. M. Kumar, Deteriorating Inventory Model With Controllable Deterioration Rate For Time- Dependent Demand and Time-Varrying Holding Cost, Yugoslav Journal of Operations Research. 24 (2014), Issue 1, 87-98.

[36] V. M. Kumar, Deteriorating Inventory Model Using Preservation Technology with Salvage Value and Shortages, Advances in Production Engineering \& Management, 8 (3) (2014), 185-192.

[37] Y. Yang, H. Chi, O. Tang, W. Zhou and T. Fan, Cross Perishable Effect on Optimal Inventory Preservation Control, European Journal of Operational Research, 276 (2018), 9981012.

[38] Y. P Lee and C. Y Dye, An inventory model for deteriorating items under stock-dependent demand and controllable deterioration rate, Computers \& Industrial Engineering, 63 (2) (2012), 474-482.

[39] Y. He and H. Huang, "Optimizing Inventory and Pricing Policy for Seasonal Deteriorating Products with Preservation Technology Investment," Journal of Industrial Engineering, Article ID 793568, 7 (2013).

[40] Z. Tao, Z. Zhang, D. Peng, Y. Shi and Y. Shi, Joint Advertising and Preservation Service Decision in a Supply Chain of Perishable Products with Retailer's Fairness Concerns. 11th CIRP Conference on Industrial ProductService Systems, Procedia CIRP 83 (2019), 461-466. 